\title{
Double Kinetics of Intermetallic Phase Precipitation in UNS S32205 Duplex Stainless Steels Submitted to Isothermal Heat Treatment
}

\author{
Igor Jordão Marques ${ }^{a}$, André de Albuquerque Vicente ${ }^{b}$, Jorge Alberto Soares Tenório ${ }^{b}$,
}

Tiago Felipe de Abreu Santos *

\author{
${ }^{a}$ Department of Mechanical Engineering, Universidade Federal de Pernambuco, Av. da Arquitetura, \\ $s / n$, Cidade Universitária, Recife, PE, Brazil \\ ${ }^{b}$ Department of Chemical Engineering, Universidade de São Paulo, Rua do Lago, 250, Cidade \\ Universitária, São Paulo, SP, Brazil
}

Received: December 12, 2016; Revised: March 16, 2017; Accepted: May 21, 2017

\begin{abstract}
One of the most studied deleterious phases in stainless steels is the sigma phase, due to its high potential to decrease the toughness and corrosion resistance of these steels. Eight samples of as-received cold rolled UNS S32205 duplex stainless steel were submitted to isothermal heat treatments at 850 ${ }^{\circ} \mathrm{C}$ during $10,15,30,60,120,180,240$, and 300 minutes in order to study the precipitation kinetics of the sigma and chi deleterious phases. Several complementary microstructural analysis techniques were used to determine the volume fraction of the intermetallic phases, including optical microscopy and scanning electron microscopy (SEM) coupled with energy dispersive spectroscopy (EDS). Evaluation was made of the kinetics of isothermal formation of sigma and chi phases in commercial duplex stainless steels (UNS S32205). The results indicated two different mechanisms for sigma phase precipitation: sigma phase formed from the chi phase for shorter isothermal heat treatment times, and sigma phase precipitated at the ferrite-austenite interface for longer isothermal heat treatment times, using a temperature of $850^{\circ} \mathrm{C}$. The phase transformation kinetics determined using the JMA equation indicated that chi phase precipitation caused faster sigma phase formation.
\end{abstract}

Keywords: Duplex stainless steels, sigma phase, chi phase, isothermal heat treatment

\section{Introduction}

Duplex stainless steels are used in applications requiring high levels of mechanical strength and corrosion resistance. The microstructures of these steels consist of balanced volume fractions of austenite and ferrite. However, the presence of ferrite stabilizers, such as chromium and molybdenum, favors the precipitation of deleterious intermetallic phases, which once formed reduce the solubilized chromium content in the matrix, severely reducing the corrosion resistance of these steels, and also lead to reduced ductility ${ }^{1,2}$. Among the deleterious phases that precipitate at high temperatures in duplex stainless steels, the sigma and chi phases are especially relevant. The sigma phase $\mathrm{Fe}$-Cr intermetallic phase is the most studied deleterious phase in stainless steels, because the exposure of these steels to high temperatures causes substantial precipitation of this phase, resulting in high volumetric fractions of sigma phase in the microstructure of the steel. The chi phase presents lower precipitation fractions and studies have shown that its precipitation is associated with sigma phase formation ${ }^{1,3}$.

The precipitation of the sigma and chi phases occurs in the temperature ranges $600-1000^{\circ} \mathrm{C}$ and $700-950^{\circ} \mathrm{C}$, respectively ${ }^{1,4-6}$, and the precipitation sites are at the $\alpha / \gamma$ (ferrite/austenite) interfaces. Chi phase can precipitate at the $\alpha / \alpha$ interface, and both sigma and chi phases also precipitate at triple $\alpha / \alpha / \gamma$ points. Studies

\footnotetext{
* e-mail: tiago.felipe@ufpe.br
}

have proposed that the precipitation of hexagonal chromium nitride $\left(\mathrm{Cr}_{2} \mathrm{~N}\right)$, which precedes the precipitation of chi and sigma phases, favors the precipitation of these deleterious intermetallic phases ${ }^{6-8}$. The precipitation of the sigma phase occurs according to a eutectoid reaction in which a secondary austenite with low chromium content precipitates. Secondary austenite is formed due to the increase of the content of austenite stabilizers $(\mathrm{Ni}$, $\mathrm{N}$, and $\mathrm{Mn}$ ) and chromium depletion in the region around the sigma phase. As the secondary austenite has lower chromium content, its presence close to the sigma phase controls the formation of sigma. However, this secondary austenite can also be considered a deleterious phase because it presents low corrosion resistance ${ }^{7}$. The kinetics of phase transformations in heterogeneous materials such as duplex stainless steel can be determined using the Johnson-Mehl-Avrami (JMA) model ${ }^{8,9}$, described by Equation 1:

$$
f=1-e^{-(K t)^{n}}
$$

where $f$ indicates the phase volumetric fraction transformed in time $t ; K$ is the Avrami time constant, which is related to the temperature and activation energy of the process; and $n$ is the Avrami exponent, which is related to the form that the precipitated phase acquires during the increase of its volume fraction. Higher values of $n$ indicate the formation of more 
acicular phases and rapid depletion of nucleation sites. Lower values of $n$ reflect the formation of equiaxed morphology phases and indicate that the nucleation process continues to occur even in advanced stages of transformation ${ }^{10}$.

The aim of this investigation was to evaluate the double kinetics and synergetic behaviors of the chi and sigma phases present in UNS S32205 duplex stainless steel following isothermal treatments. A final annealing was performed after the manufacturing steps, but the rolling structure nonetheless altered the intermetallic kinetics, as found here when the steel was submitted to isothermal treatments. As the material is used in this form, it is important to investigate the precipitation of deleterious secondary phases in order to ensure satisfactory properties of the duplex stainless steel.

\section{Experimental Procedure}

A cold rolled and solution-treated $6 \mathrm{~mm}$-thick plate of UNS S32205 duplex stainless steel was used. The chemical composition of the material is shown in Table 1. The plate was sectioned into samples that were subjected to isothermal heat treatments in a muffle furnace at $850{ }^{\circ} \mathrm{C}$, in an air atmosphere, for different time intervals between 10 minutes and 5 hours. At the end of the isothermal heat treatment, the sample was withdrawn and rapidly cooled in water in order to preserve the high-temperature microstructure developed during the heat treatment. After the isothermal heat treatments, the samples were submitted to metallographic preparation, which consisted of grinding with 220 , 320,600 , and 1200 mesh papers, followed by polishing with 0.3 and $0.1 \mu \mathrm{m}$ diamond abrasives. The samples were then analyzed by optical microscopy and scanning electron microscopy. The samples were analyzed without etching and with electrochemical attack in $10 \% \mathrm{KOH}$ solution for 60 seconds at $2.5 \mathrm{~V}$. The purpose of this etching was to reveal the sigma and chi phases.

Quantitative stereological analysis of the microscopy images was performed using Image $J$ software, quantifying the area fraction filled by precipitates, the average area of the precipitates, and their circularity. Figure 1 illustrates the determination of the circularity.

\section{Results and Discussion}

\subsection{Qualitative analysis}

Figure 2 shows the evolution of the intermetallic phase precipitation for different times of isothermal heat treatment at $850{ }^{\circ} \mathrm{C}$. Scanning electron microscopy analysis of the samples submitted to shorter isothermal heat treatment and etched by $\mathrm{KOH}$ showed the formation of small circular intermetallic phases at the $\alpha / \gamma$ interfaces. As the isothermal heat treatment time increased, there was growth of these phases, following the $\alpha / \gamma$ interface. For isothermal heat treatment times of over 1 hour, the intermetallic phases started to grow towards ferrite, evidencing the consumption of ferrite for the precipitation of these deleterious intermetallic phases. There was also an increase in the number of sites of secondary austenite, because the precipitation of the sigma phase entailed the formation of secondary austenite depleted in chromium. Analysis of the samples submitted to shorter isothermal heat treatments showed that at the beginning of the transformation, the intermetallic phases presented lamellar morphology intermediate between austenite and ferrite. As the transformation proceeded, these intermetallic phases tended to become equiaxed.

Scanning electron microscopy analysis was used to distinguish between the intermetallic phases in the non etched samples using the backscattered electron signal. Figure 3 shows the chemical composition spectra obtained in the EDS analyses. Table 2 shows the results of the EDS chemical composition analysis for points of each phase. Four different phases were identified from the EDS spectra. Comparison with results reported in the literature confirmed that the precipitates corresponded to the sigma and chi phases, with much higher levels of Mo in the chi phase $e^{6,11}$. The distinction between austenite and ferrite was based on the difference in the Ni contents of these phases.

The EDS analyses showed that both the sigma $(\sigma)$ and chi $(\chi)$ intermetallic phases presented lower contents of iron and higher contents of chromium, compared to the austenite

Table 1. Chemical composition (wt.\%) of UNS S32205 duplex stainless steel.

\begin{tabular}{lcccccccccc}
\hline UNS & $\mathrm{C}$ & $\mathrm{Si}$ & $\mathrm{Mn}$ & $\mathrm{Cr}$ & $\mathrm{Ni}$ & $\mathrm{Mo}$ & $\mathrm{N}$ & $\mathrm{P}$ & $\mathrm{S}$ \\
\hline $\mathrm{S} 32205$ & 0.02 & 0.30 & 1.80 & 22.50 & 5.40 & 2.80 & 0.16 & 0.030 & 0.001 \\
\hline
\end{tabular}

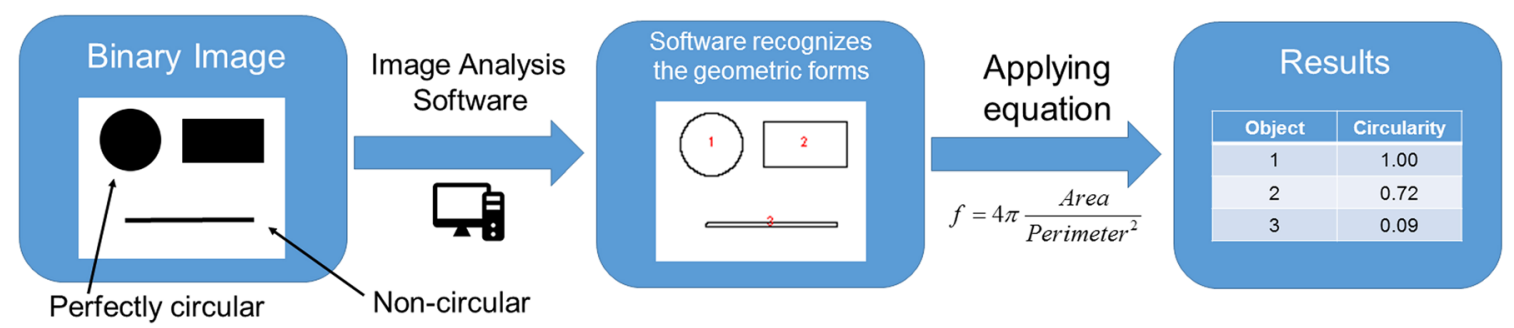

Figure 1. Schematic procedure for determination of circularity. 


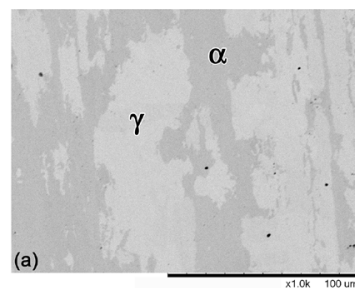

(b)
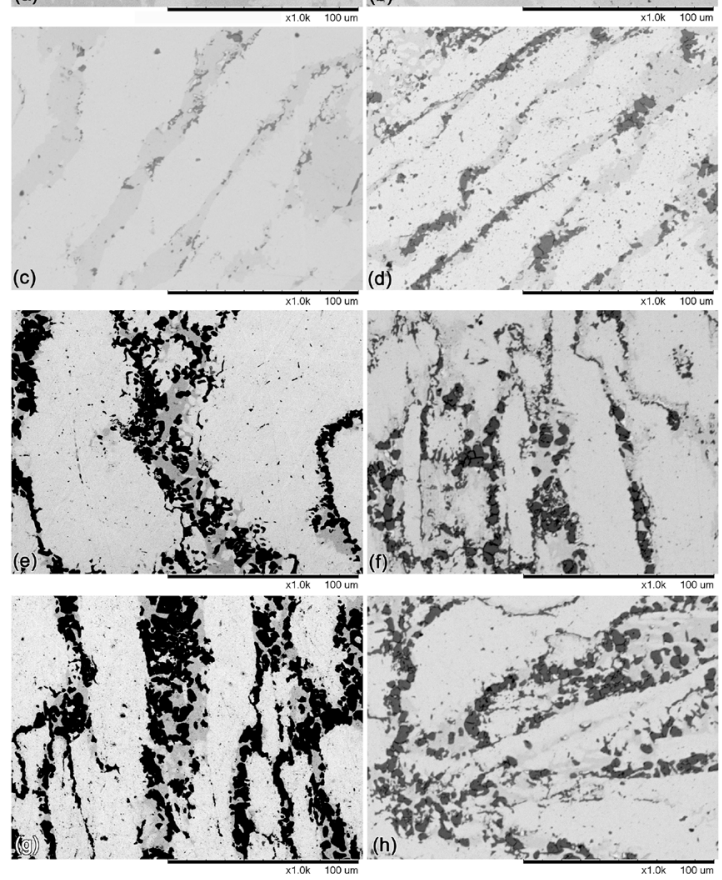

Figure 2. SEM micrographs showing the precipitation of intermetallic phases for different durations of isothermal heat treatment at 850 ${ }^{\circ} \mathrm{C}$. (a) Non-aged, (b) 10 minutes, (c) 30 minutes, (d) 1 hour, (e) 2 hours, (f) 3 hours, (g) 4 hours, and (h) 5 hours.

$(\gamma)$ and ferrite $(\alpha)$. The austenite $(\gamma)$ presented the highest content of nickel, while the $\chi$ phase contained much more molybdenum than the other phases.

Figure 4 shows SEM backscattered electron micrographs for different isothermal heat treatment times. The backscattered electron SEM signal can distinguish phases with different chemical composition, since the electron backscattering is more intense for heavier chemical elements. A heavier phase appears brighter, while a lighter phase appears darker. In Figure 3, the chi phase is heavier (brighter) than the sigma phase, followed by austenite and finally ferrite (darker), reflecting the chemical composition (Table 2). The backscattered SEM data indicated that for very short transformation times (up to about 15 minutes), the chi phase constituted the largest volumetric fraction of the precipitated intermetallic phases. However, the sigma phase rapidly became the largest volumetric fraction of these phases. For isothermal heat treatments at $850^{\circ} \mathrm{C}$ shorter than 1 hour, the sigma phase already corresponded to more than $90 \%$ of the volumetric fraction of the precipitated intermetallic phases.
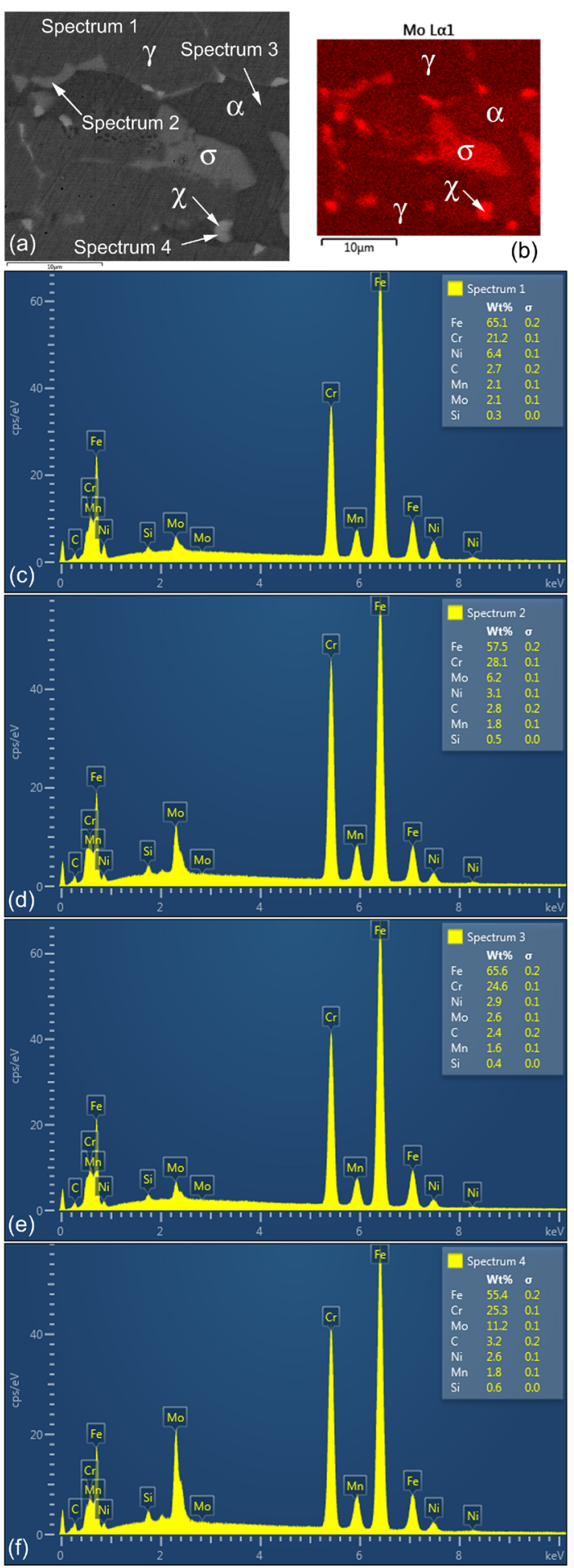

Figure 3. Map of chemical composition obtained by EDS for a sample submitted to 30 minutes isothermal heat treatment. (a) SEM micrograph analysis showing five different phases; (b) EDS spectrum 1: $\gamma$ (austenite); (c) EDS spectrum 2: $\sigma$ (sigma phase); (d) EDS spectrum 3: $\alpha$ (ferrite); (e) EDS spectrum 4: $\chi$ (chi); (f) EDS spectrum 5: $\chi$ (chi). 
Table 2. Chemical compositions of the $\gamma, \alpha, \sigma$, and $\chi$ phases quantified by EDS.

\begin{tabular}{lcccccc}
\hline \multirow{2}{*}{ EDS spectrum } & Phase & $\mathrm{Fe}$ & $\mathrm{Cr}$ & $\mathrm{Ni}$ & $\mathrm{Mo}$ & $\mathrm{Mn}$ \\
\hline 1 & $\gamma$ & 65.1 & 21.2 & 6.4 & 2.1 & 2.1 \\
2 & $\sigma$ & 57.5 & 28.1 & 3.1 & 6.2 & 1.8 \\
3 & $\alpha$ & 65.6 & 24.6 & 2.9 & 2.6 & 1.6 \\
4 & $\chi$ & 55.4 & 25.3 & 2.6 & 11.2 & 1.8 \\
5 & $\chi$ & 55.6 & 24.7 & 3.1 & 10.9 & 1.9 \\
\hline
\end{tabular}
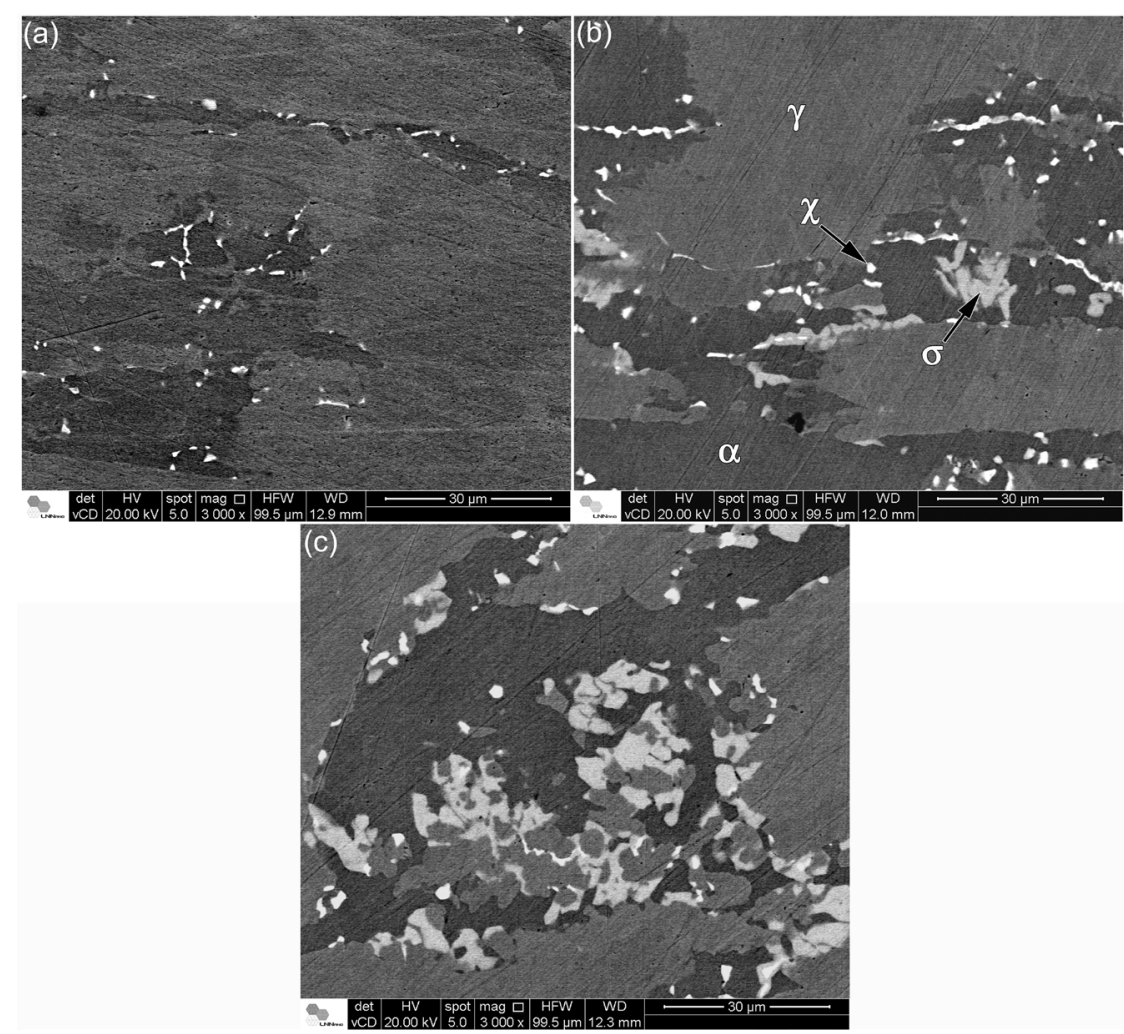

Figure 4. Non-etched micrographs obtained using the SEM backscattered electron signal for different durations of isothermal heat treatment at $850^{\circ} \mathrm{C}$. (a) 10 minutes, (b) 30 minutes, (c) 60 minutes.

\subsection{Quantitative analysis}

A quantitative stereological analysis was performed to determine the area fraction occupied by the precipitates, the average area of the precipitates, and the circularity of the intermetallic precipitates. The stereological analysis was based on ASTM E1245-03. Twenty fields of each sample were analyzed and Image J software was used for quantification. Table 3 shows the mean volume fractions occupied by the intermetallic phases, for each treatment time, obtained from the SEM images. As expected, there was an increase in the intermetallic phase volumetric fraction, which became asymptotic for longer treatment times, in agreement with the Avrami kinetics 8
The circularity is a form factor, described by Equation 2 , whose value varies between 0 and 1 , with 0 indicating perfectly acicular particles and 1 corresponding to perfectly circular precipitates ${ }^{13}$.

$$
f=4 \pi \frac{\text { Area }}{\text { Perimeter }^{2}}
$$

At the start of the transformation, there was the formation of small spherical nuclei, which explained the high circularity values obtained for the first stages of precipitation. However, with growth of the precipitates following the $\alpha / \gamma$ interfaces, there was a rapid reduction of circularity, followed by 
Table 3. Volume fractions of intermetallic phase obtained from Image $J$ analysis.

\begin{tabular}{lcccc}
\hline Heat treatment & Time & Time $(\mathrm{s})$ & Volume fraction of sigma phase (\%) & $95 \%$ CI \\
\hline 1 & $10 \mathrm{~min}$ & 600 & 2.1 & 0.6 \\
2 & $15 \mathrm{~min}$ & 900 & 2.8 & 0.3 \\
3 & $30 \mathrm{~min}$ & 1800 & 4.7 & 0.4 \\
4 & $1 \mathrm{~h}$ & 3600 & 15 & 1 \\
5 & $2 \mathrm{~h}$ & 7200 & 18 & 1 \\
6 & $3 \mathrm{~h}$ & 10800 & 21 & 2 \\
7 & $4 \mathrm{~h}$ & 14400 & 22 & 1 \\
8 & $5 \mathrm{~h}$ & 18000 & 23 & 1 \\
\hline
\end{tabular}

stabilization of the values for isothermal heat treatment times longer than 1 hour.

Figure 5 shows the evolution of the circularity and the mean area of the intermetallic phase precipitates. The change in circularity corroborated the change in precipitation of intermetallic phases indicated by the qualitative analysis, since the temporal derivative of the circularity remained almost constant after 1 hour of isothermal treatment. However, the mean volume fraction of the precipitates presented higher growth up to two hours of isothermal heat treatment at 850 ${ }^{\circ} \mathrm{C}$, followed by an asymptotic trend for longer times.

The experimental volumetric fraction data were plotted using the linearized form of the JMA equation (Equation 3), from which the change in the precipitation behavior of the phases could be observed, in agreement with the qualitative analyses and the evolution of the circularity.

$\ln [-\ln [1-y(t)]]=n \ln (t)+n \ln (K)$

Figure 6 shows the results of the linear fitting of the experimental data, described by Equations 4 and 5, respectively, for the intermetallic phases formed during heat treatment for time $t$ in the early and advanced stages of transformation. The experimental intermetallic volumetric fraction data were fitted to the JMA model using two linear adjustments: one for the beginning of transformation and the other for more advanced stages of precipitation. The value of the Avrami exponent for the first stages of precipitation was higher than for the last stage of precipitation, suggesting a greater tendency for diffusion across the grain boundary (also called interface controlled growth). In the advanced stage, the smaller value of $n$ suggested that diffusion through grain volume was the main process controlling the transformation ${ }^{9,11}$. This stage involved the growth of the intermetallic precipitates towards the ferrite $(\alpha)$ grains.

$$
\begin{aligned}
& y(t)=1-e^{-\left(8.33 \times 10^{-6} \times t\right)^{0.73}} \\
& y(t)=1-e^{-\left(2.62 \times 10^{-7} \times t\right)^{0.25}}
\end{aligned}
$$

The SEM analysis with images generated by backscattered electrons also indicated that the second kinetic model corresponded to precipitation of the sigma phase alone, while the first kinetic model corresponded to the precipitation of both chi and sigma phases.

Considering the trend line of the kinetic fit for advanced isothermal heat treatment times, it is clear that if this second kinetics occurred at the beginning of the transformation,
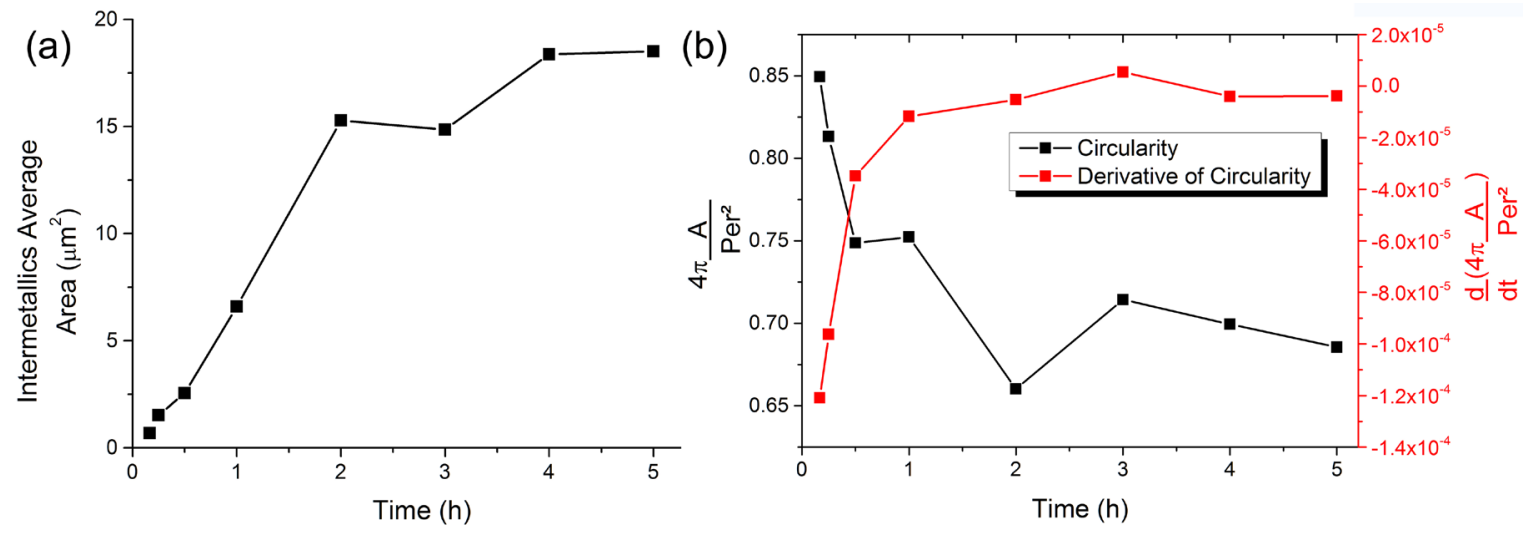

Figure 5. Phase transformations according to time during isothermal heat treatment at $850{ }^{\circ} \mathrm{C}$. (a) circularity of the intermetallic phase precipitates, (b) mean area of the intermetallic phase precipitates. 


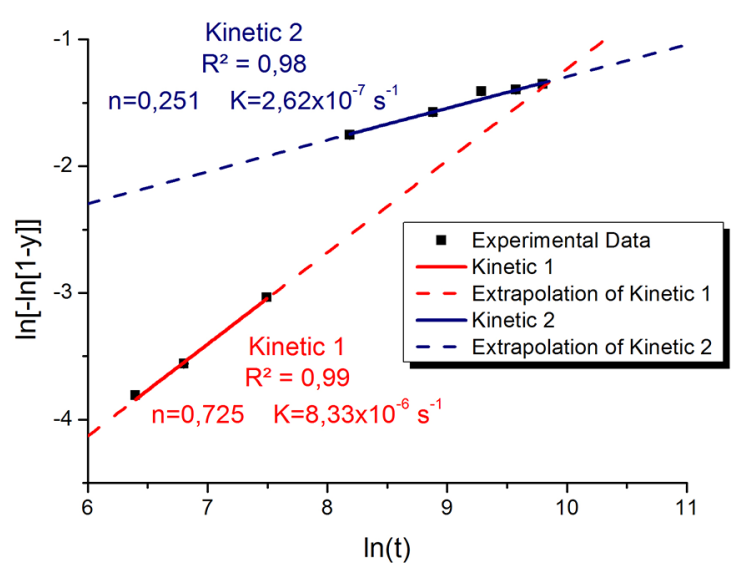

Figure 6. Linear fits of the experimental sigma phase volumetric fraction data obtained for each time of isothermal heat treatment at $850^{\circ} \mathrm{C}$.

there would be a greater volumetric fraction of sigma phase at the beginning of the transformation, which was not observed for shorter times of isothermal heat treatment at $850^{\circ} \mathrm{C}$. The fact that the sigma phase volume fraction at the beginning was much lower than that indicated by the trend line provided strong evidence that formation of the chi phase was an important step for subsequent formation of the sigma phase.

The analysis using the backscattered electron SEM signal showed that the sigma phase frequently interfaced with the chi phase precipitates, indicating that, at first, the chi phase interface acted as the preferred site for sigma phase nucleation. Nucleation and growth of the chi phase was inhibited by the presence of the sigma phase at its interface and the reduction of its possible nucleation sites, which provided an explanation for the rapid stabilization of the chi phase volume fraction. In addition, it has been reported that since the chi phase is surrounded by sigma phase, it is consumed by the formation of the sigma phase ${ }^{3}$.

Previous studies ${ }^{12,14}$ have suggested the existence of double kinetics for the precipitation of the sigma phase in duplex stainless steels, but with the non-occurrence of double kinetics for some temperatures. Elmer et al. ${ }^{14}$ did not observe double kinetics for formation of the sigma phase at $700{ }^{\circ} \mathrm{C}$. Since this temperature is at the lower limit of the chi phase formation temperature range, it is possible that chi phase precipitation did not occur in this experiment.

Dos Santos et al. ${ }^{12}$ identified double kinetics of sigma phase formation at temperatures between $700{ }^{\circ} \mathrm{C}$ and 900 ${ }^{\circ} \mathrm{C}$, with simple kinetics identified at $950{ }^{\circ} \mathrm{C}$. In this work, however, the presence of the chi phase at temperatures between $850{ }^{\circ} \mathrm{C}$ and $950{ }^{\circ} \mathrm{C}$ was not identified. This behavior could be attributed to the solubilization treatment before isothermal aging, which would cause the development of different microstructural conditions (grain size, and different morphology of the austenite and ferrite clusters), leading to changes in the kinetics ${ }^{7}$ and favoring rapid consumption of the chi phase. Wong et al. ${ }^{15}$ showed that dissolution of the sigma phase could occur at 860 to $910{ }^{\circ} \mathrm{C}$ and at 910 to $930{ }^{\circ} \mathrm{C}$ under continuous heating. This temperature range is dependent on the sigma phase volume fraction, so some competition between dissolution and sigma phase formation may disguise double kinetics in the isothermal transformation at $950{ }^{\circ} \mathrm{C}$.

Other works that have investigated the kinetics of sigma phase precipitation, including simple kinetics, have evaluated the transformation over longer periods, when the sigma phase is predominant. Differences in the solution-treated state of the non-aged metal alter the duration of each transformation mechanism, due to changes in the initial grain size and the dislocations density of the base metal. The initial solutiontreated condition of the base metal plays a very important role in the kinetic behavior of the precipitation ${ }^{7,16}$.

\section{Conclusions}

Precipitation of the deleterious intermetallic sigma and chi phases in as-rolled UNS S32205 duplex stainless steel was observed during isothermal heat treatments at $850^{\circ} \mathrm{C}$. The precipitation of the intermetallic phases preferentially followed the $\alpha / \gamma$ interface, occurring later in the direction of the ferrite grains as the $\alpha / \gamma$ interface was consumed. The circularity of the intermetallic phases and the average area of the precipitates showed asymptotic behavior from at least 3 hours. Based on the JMA model, it could be concluded that the formation of the sigma phase involved double kinetics. The first stage was strongly influenced by the presence of the chi phase as nucleation sites, while in the second stage the growth of the precipitates was mainly towards the ferrite grains.

\section{Acknowledgments}

Financial support for this work was provided by CAPES, CNPq, UFPE, and FACEPE. The authors are grateful to LNNano / CNPEM / MCTI for the use of infrastructure and equipment, and to Aperam South America for donation of the material.

\section{References}

1. Lo KH, Shek CH, Lai JKL. Recent developments in stainless steels. Materials Science and Engineering: R: Reports. 2009;65(4-6):39-104.

2. Sorensen CD, Nelson TW. Sigma phase formation in friction stirring of iron-nickel-chromium alloys. In: $7^{\text {th }}$ International Conference on Trends in Welding Research; 2005 May 16-20; Pine Mountain, GA, USA. p. 441-446.

3. Escriba DM, Materna-Morris E, Plaut RL, Padilha AF. Chiphase precipitation in a duplex stainless steel. Materials Characterization. 2009;60(11):1214-1219. 
4. Voronenko BI. Austenitic-ferritic stainless steels: A state-of-the-art review. Materials Science and Heat Treatment. 1997;39(10):428-437.

5. Lippold JC, Kotechi DJ. Welding Metallurgy and Weldability of Stainless Steels. Hoboken: Wiley; 2005. 376 p.

6. Pohl M, Storz O, Glogowski T. $\sigma$-phase morphologies and their effect on mechanical properties of duplex stainless steels. International Journal of Materials Research. 2008;99(10):1163-1170.

7. Llorca-Isern N, López-Luque H, López-Jimenez I, Biezma MV. Identification of sigma and chi phases in duplex stainless steels. Materials Characterization. 2016;112:20-29.

8. Pardal JM, Tavares SSM, Fonseca MPC, Souza JA, Vieira LM, Abreu HFG. Deleterious phases precipitation on superduplex stainless steel UNS S32750: characterization by light optical and scanning electron microscopy. Materials Research. 2010;13(3):401-407.

9. Avrami M. Kinetics of Phase Change. I General Theory. The Journal of Chemical Physics. 1939;7(12):1103-1112.

10. Johnson WA, Mehl RF. Reaction kinetics in processes of nucleation and growth. Transactions of The Metallurgical Society of AIME. 1939;135:416-442.
11. Christian JW. Formal Theory of Transformation Kinetics. In: Christian JW. The Theory of Transformations in Metals and Alloys. Oxford: Pergamon; 2002. p. 529-552.

12. dos Santos DC, Magnabosco R. Kinectic Study to Predict Sigma Phase Transformation in Duplex Stainless Steels. Metallurgical and Materials Transactions A. 2016;47(4):1554-1565.

13. Pohl M, Storz O, Glogowski T. Effect of intermetallic precipitations on the properties of duplex stainless steel. Materials Characterization. 2007;58(1):65-71.

14. Elmer JW, Palmer TA, Specht, ED. Direct observations of sigma phase formation in duplex stainless steels using in-situ synchrotron X-ray diffraction. Metallurgial and Materials Transactions A. 2007;38(3):464-475.

15. Wong, KW, Shek CH, Zhang W, Lai JKL. $\sigma$ phase dissolution in duplex stainless steel at elevated temperature studied by thermal analysis. Materials Letters. 2008;62(24):3991-3994.

16. Magnabosco R. Kinetics of sigma phase formation in a Duplex Stainless Steel. Materials Research. 2009;12(3):321-327. 